\title{
PHASE DIAGRAM OF GELATINE-POLYURONATE COLLOIDS: ITS APPLICATION FOR MICROENCAPSULATION AND NOT ONLY
}

\author{
Alexei Baerle*, Olga Dimova, Irina Urumoglova, Pavel Tatarov, Larisa Zadorojnai \\ Technical University of Moldova, 168, Stefan cel Mare Blvd., Chisinau MD-2004, Republic of Moldova \\ *e-mail: abaerle2003@mail.ru; phone: (+373 79) 7893 01; fax: (+373 22) 509960
}

\begin{abstract}
Phase state and the charge of colloidal particles in the gelatine-polyuronate system were studied. A method for comparative evaluation of molecular weight of colloids by means of viscosimetric measurements and electrophoresis was developed. It is shown that the Diagram $\{$ Phase state $=\mathrm{f}$ (composition, $\mathrm{pH})\}$ contains six welldefined regions. The diagram explains and predicts the behaviour of protein-polysaccharide colloids, which are included in beverages or forms the shells of oil-containing microcapsules.
\end{abstract}

Keywords: protein-polysaccharides, colloids, electrokinetic potential, phase diagram, microcapsules.

Received: March 2016/ Revised final: April 2016/Accepted: April 2016

\section{Introduction}

Poorly soluble or electro-statically agglomerated macromolecular compounds derive from interaction of protein zwitterions with polyanions, in particular, with polyuronic acids and their salts [1]. These interactions are of great practical interest, insofar as much of foods contain biopolymers in different combinations [2]. Recently, there were published many papers devoted to the joint use of the proteins and polysaccharides, originate from different natural raw materials, in various pharmaceutical and food systems [3-6]. These compounds have a greater ability to precipitate the colloidal suspensions, compared to pure linear polymers [7]. It is believed that these mixtures are in fact the covalent chemical compounds or supra-molecular three-dimensional structures with high molecular weight, charged as zwitterions due to the presence of proteins [8-10]. Due to this fact, the mixtures are capable to precipitate not only the neutral particles, but also charged particles, both positive and negative. Such three-dimensional structures (3D) appear also during the complex coacervation of oppositely charged macromolecules on the oil droplets surface in the microencapsulation processes [11]. The optimum conditions of precipitation of alginate-gelatine coacervates were obtained in vitro, using measurements of turbidity and viscosity [1].

The study led to the elaboration of different phase diagrams, which reflects complex interactions between the negatively charged polysaccharides and zwitterions of proteins $[8,12,13]$. The interaction of proteins with polysaccharides was described in numerous research papers, but not in the exhaustive form. Among the problematic issues it can be identified the determination of the molecular weight of the resulting supramolecular structures. In our view, the estimation of the molecular weights will be useful for calculating of the microcapsule shells parameters, as well as to determine the conditions of deposition of neutral colloidal particles. Detailed analysis of the phase state of the gelatine-polyuronate system is important to determine the optimal sedimentation conditions, or opposite, to prevent the coagulation of proteins by small amounts of extraneous poly-anions. In constructing of the phase diagram of the gelatine-polyuronate, it is important to consider the sign and magnitude of charge of generated complexes. Unambiguous information about the charge of colloidal particles is given by method of electrophoresis, allowing to measure the zeta potential and to fractionate the charged particles $[14,15]$. Values of charges are important for explanation and prognosis of the colloids behaviour in food systems containing proteins and polysaccharides. This equally applies to colloids used for the microencapsulation of biologically active substances $[4,8,11]$.

\section{Experimental part \\ Preparation of gelatine solutions}

Macromolecular complexes were prepared using air-dried instant soluble food gelatine of premium grade (Lisichansk Gelatin Factory, ALC). All gelatine samples were incubated in a bath with boiling water for at least 5 minutes with continuous stirring until their complete dissolution. The native $\mathrm{pH}$ of gelatine solutions was in the range of 6.3...6.9. To adjust the desirable $\mathrm{pH}$ values, which were situated at the range of $2.5 \ldots 8.0$ units, the $\mathrm{pH}$ value was decreased by adding of small crystals of citric acid, or was increased by adding of solid potassium hydroxide. Pure gelatine solutions $(0.5 \%)$ were prepared to determine the isoelectric point (IEP) of gelatine, in order to determine and to consider its type. The gels with the highest turbidity, indicating zero charge of macromolecules, were obtained after $24 \mathrm{~h}$ in the vials with $\mathrm{pH}$ value ranged from 4.7 to 4.9 . These values correspond to IEP of the gelatine type "B", equal to 4.6...5.4, usually obtained by alkaline hydrolysis of skins [12].

Preparation of polyuronates

Pure preparations of alginic acid and hyaluronic acid were isolated from thallus of Laminaria Japonica [16] 
and from cockscombs of Gallus Domesticus [17], respectively. Potassium salts were prepared by dropwise addition of $\mathrm{KOH}$ into suspensions of corresponding acids, up to their complete dissolution at $\mathrm{pH}=5.5 \ldots 7.5$. Potassium alginate has been used as the main polyuronate, because of the possibility to separate it in pure form in situ [16]. Preparations of hyaluronic acid cannot be considered as "pure", because of inevitable presence of protein traces [9, 17]. Due to this fact, the salts of hyaluronic acid have been used to prepare coacervate microcapsules, in order to verify the applicability of results to similar systems, not only to gelatine-alginate system.

\section{Specific viscosity and viscosimetric molecular weights}

Viscosimetric measurements of high molecular compounds (HMC) were determined by viscosimeter "VPJ-1-0.34" (Apparaturshhik Co., Russia).

\section{Preparation of colloids}

The gelatine-polyuronate colloidal systems only by leisurely addition of polyuronate solutions in the gelatine, never vice versa, were prepared. Combination of biopolymers was accompanied by vigorous stirring, and after that, the colloids were subjected to analysis immediately.

\section{Oil-containing microcapsules}

The microcapsules, containing walnut oil or sunflower oil, with gelatine-alginate or gelatine-hyaluronate shells, were obtained by complex coacervation method [18]. The ratio of gelatine/polyuranate in the shells of microcapsules was of 3.0..6.0. Mass fraction of encapsulated oil was no less than $75 \%$. Their sizes were ranged from 5.0 to 50.0 microns. Estimated thickness of shells was approx. 0.38 microns.

\section{Electrokinetic potential}

Zeta potential ( $\zeta$ ) of colloids was measured by simply device (Figure 1).
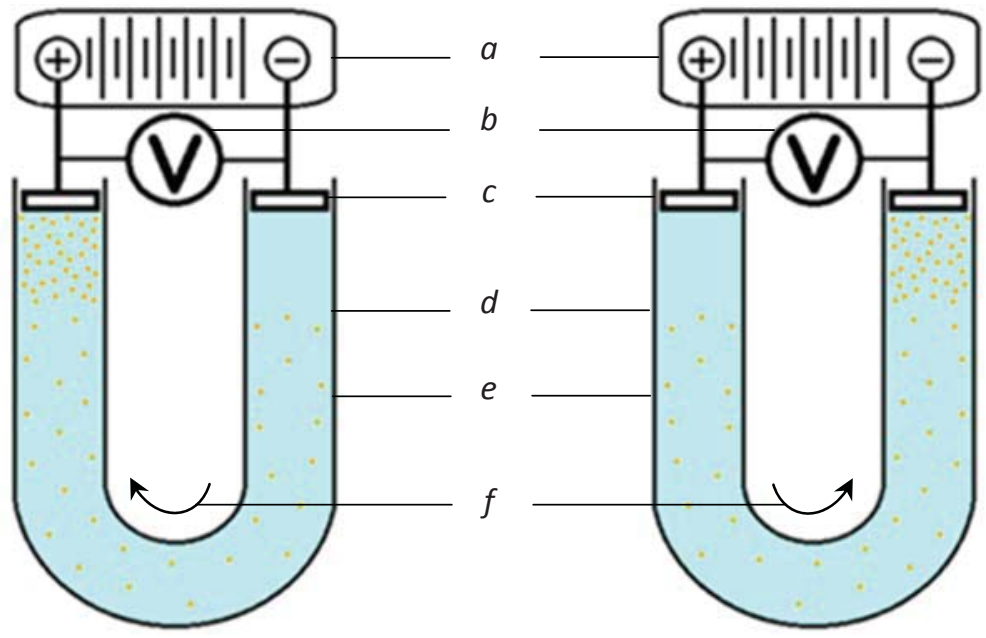

Figure 1. Electrophoretic device: a) source of voltage; b) digital multi-tester; c) stainless steel electrodes; d) sol border; e) U-shaped tube; f) movement of "_"charged particles (on left), "+"charged (right).

Electrophoresis was performed in U-shaped tubes of $1.0 \mathrm{~cm}$ width. Process has been occurred on the surface of hand-made integral electrodes, confectioned from stainless steel AISI 304 ("inox" for cookware). The distance between surfaces of the electrodes was from 15.0 to $25.0 \mathrm{~cm}$. The field strength values (E) were adjusted in the range of $100 \ldots 400 \mathrm{~V} / \mathrm{m}$ by Stand for Testing of Automatically Equipment (STAE) "SPA-97” (Contragent Co., Ukraine). Operating voltage has been monitored by means of Digital Multi-tester "UT33C" (Shenzhen Sunkoo-Reid Electronic Co., China). The $\zeta$-potential was calculated by the Helmholtz-Smoluchowski equation, Eq.(1) [7,14,15]:

$\zeta=\frac{\eta \cdot V}{\varepsilon \cdot \varepsilon_{0} \cdot H}=\frac{\eta \cdot l \cdot L}{\varepsilon \cdot \varepsilon_{0} \cdot U \cdot \tau}$

where, constants: $\eta=1 \cdot 10^{-3}(\mathrm{~Pa}) ; \cdot s$-the viscosity of water; $\varepsilon=89$, non-dimensional - dielectric constant of water; $\varepsilon_{0}=8.85 \cdot 10^{-12}(\mathrm{~F} / \mathrm{m})$ - electric permittivity of vacuum; variables: $V(\mathrm{~m} / \mathrm{s})$ - velocity of colloid particles; $H(\mathrm{~V} / \mathrm{m})$ - intensity of electric field; $l(\mathrm{~m})$ - run of colloidal particles in the electric field; $\tau(\mathrm{s})$ - time of electrophoresis; $L(\mathrm{~m})$ - distance between surfaces of electrodes (effective tube length); $U(\mathrm{~V})$ - voltage. 


\section{Results and discussion}

\section{Molecular weights of high molecular polymeric compounds (HMCs) pure samples}

In contrast to the low molecular substances, HMCs do not have constant molecular weight. First, it is characteristic for in situ preparations of HMC, obtained from natural sources, having molecular weights that differ in function of characteristics of raw material. Thus, the molecular weights of linear biopolymers ranges from $10^{3} \mathrm{D}$ to $10^{6} \mathrm{D}(1 \ldots 1000 \mathrm{kD})[2,19]$. Secondly, the HMCs molecular weight is almost always seeming, owing to intra- and intermolecular interactions, taking place in the HMCs solutions under the influence of various physical and chemical factors [20]. This is especially characteristic for the macromolecules of proteins and polysaccharides, such as gelatine, alginate, and hyaluronate, because their properties are $\mathrm{pH}$-dependent, due to their polyionic structure, containing acidic or basic functional groups. So-called viscosimetric molecular weight of most types of polymers is usually calculated by Mark-Houwink-Sakurada equation, Eq.(2) [20,21]:

$M_{H M C}=\left(\frac{[\eta]}{K}\right)^{1 / \alpha}$

where, $K$ and $\alpha$ - constants, properly for the pair polymer-solvent; [ $\eta$ - intrinsic viscosity, which is calculated by extrapolation of function $\eta_{\text {red. }}=f\{C(H M C)\}$ to zero concentration of HMC (Figures 2 and 3 ). For calculations of $M_{H M C}$, we have considered maximum values of $[\eta]$, because of straightening of HMCs at high $\mathrm{pH}$ value [6-7].

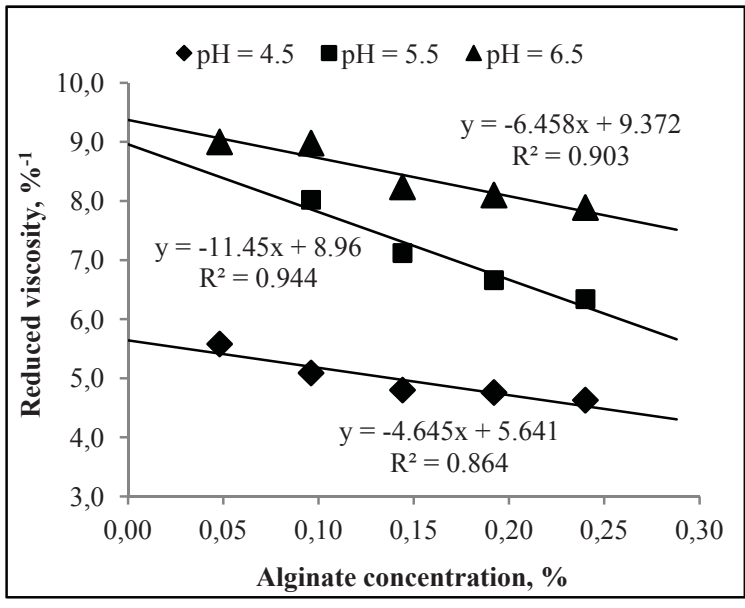

Figure 2. Viscosity of alginate.

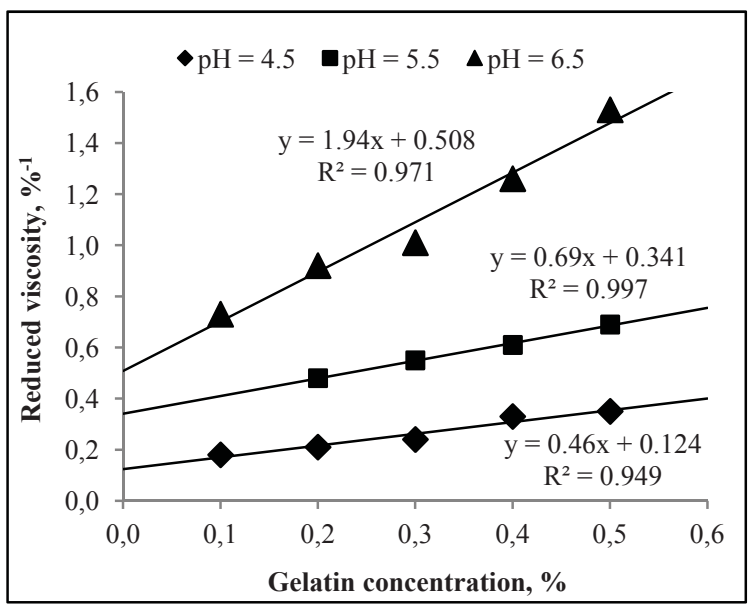

Figure 3. Viscosity of gelatine.

Samples of alginate, separated from thallus of Laminaria Japonica, possess intrinsic viscosity $[\eta]=9.37 \%{ }^{-1}$, or $937 \mathrm{~mL} / \mathrm{g}$ in other units. This value is close to intrinsic viscosity of commercial alginate (Sigma-Aldrich Corporation, USA), equal to $1040 \mathrm{~mL} / \mathrm{g}$ [21]. For this reason, respective values of $K(0.0073)$ and $\alpha(0.92)$ for commercial alginate were used to calculate molecular weight of "our" native alginate. Resulted value, $357 \mathrm{kD}$, is in good correlation with many reported results [20-22]. In case of linear proteins, as gelatine, the Mark-Houwink-Sakurada equation usually in form of Eq.(3) is written [19]:

$$
\frac{M_{\text {protein }}}{M_{0}}=\left(\frac{[\eta]}{K}\right)^{1 / \alpha}
$$

For gelatine type "B", $M_{0}$ is equal to $110 \mathrm{D}$, representing average molecular weight of structural unit of protein molecule ("average" amino acid). Experimental intrinsic viscosity of gelatine was $50.8 \mathrm{~mL} / \mathrm{g}$. Referenced values [19] of constants $K(0.166)$ and $\alpha(0.885)$ have been used to calculate the molecular weight of gelatine, being equal to $70.8 \mathrm{kD}$.

\section{Estimation of the molecular weights of colloids}

It can be assumed that the formation of gelatine-polyuronate systems is possible in a wide range of $\mathrm{pH}(2.0 \ldots 8.0)$. Extreme values of $\mathrm{pH}$ correspond to cationic form of gelatine $\left(\mathrm{Gel}^{+}\right)$and its anionic form $\left(\mathrm{Gel}^{-}\right)$, respectively. However, in the $\mathrm{pH}$ range from 8.0 to 5.0, any types of condensed (solid) colloidal systems do not form, but their interaction is not excluded. Both polymers at $\mathrm{pH}>5.0$ contribute to total (specific) viscosity of solution. For transparent mixtures of 
gelatine and polyuronic salts this dependence is different. Recall that the charge of polyuronate is an order of magnitude greater than gelatine (zwitter-ion) total charge. Due to this fact, one molecule of alginate or hyaluronate can neutralize several gelatine molecules. The analysis demonstrates that specific viscosity of mixtures depends logarithmically on ratio $m_{\mathrm{Gel}} / m_{\mathrm{Hur}}$ (Figure 4 ). This function possesses a good value of approximation accuracy, $R^{2}=0.973$. It allowed us to represent this dependence in the linear form (Figure 5). In our view, this linear relationship is well explained by a large number of charges in polyuronate molecules, being ten times higher than that of gelatine at equal $\mathrm{pH}$ values.

The weight ratio of gelatine/polyuronate allowed us to calculate the number of gelatine molecules, accompanied with one molecule of alginate, i.e. molar ratio of the biopolymers, $n_{\mathrm{Gel}} / n_{\mathrm{Alg}}$, Eq.(4):

$\frac{n_{\mathrm{Gel}}}{n_{\mathrm{Alg}}}=\frac{m_{\mathrm{Gel}}}{m_{\mathrm{Alg}}} \cdot \frac{M_{\mathrm{Alg}}}{M_{\mathrm{Gel}}}$

There were also estimated the minimal possible molecular weight of GelAlg complexes, $M_{\text {GelAlg}}$, reported to the one molecule of alginate, deducing following equation, Eq.(5):

$M_{\mathrm{GelAlg}}=M_{\mathrm{Alg}}+\frac{n_{\mathrm{Gel}}}{n_{\mathrm{Alg}}} \cdot M_{\mathrm{Gel}}=M_{\mathrm{Alg}}\left(\frac{m_{\mathrm{Gel}}}{m_{\mathrm{Alg}}}+1\right)$

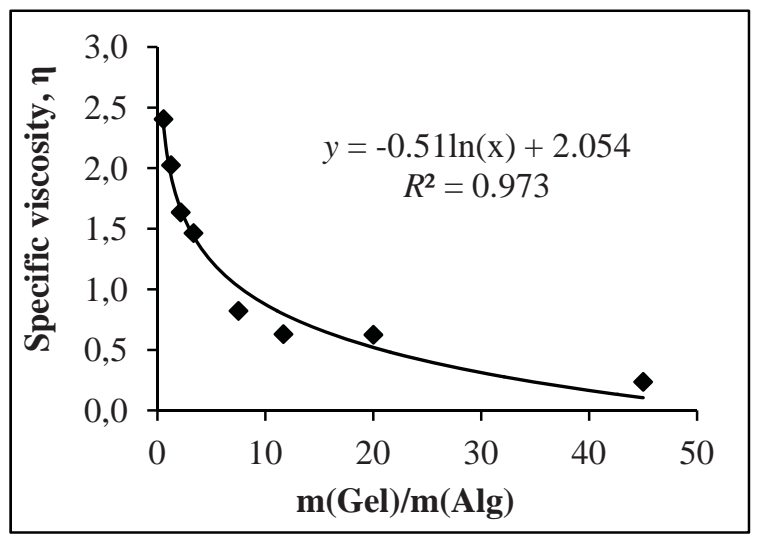

Figure 4. Specific viscosity: logarithmic function.

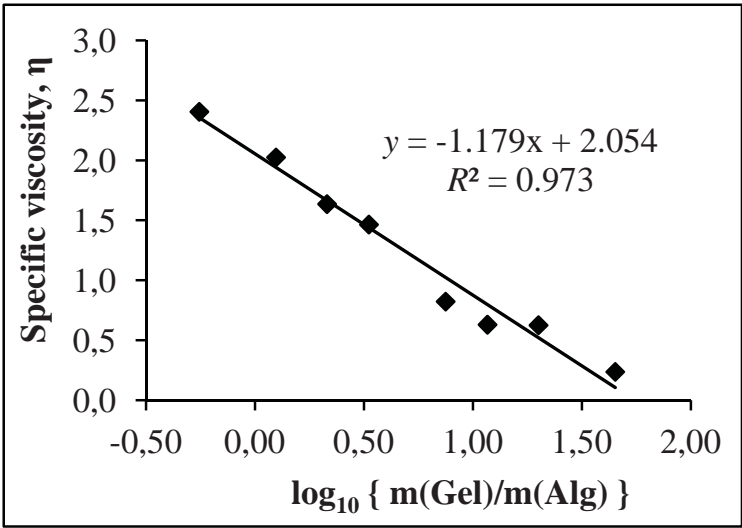

Figure 5. Specific viscosity: linear function.

At $\mathrm{pH}<$ IEP the interaction of gelatine with alginate leads to the formation of various colloids, including viscous solutions, large floccules, sols and gels. Naturally, the measuring of zeta-potential for solid colloids was possible only for the stable sols consisting from small particles, which does not precipitate immediately (Table 1).

Table 1

Type of colloidal systems and $\zeta$-potential of stable sols.

\begin{tabular}{|c|c|c|c|c|c|c|c|c|c|}
\hline$m_{G e} / m_{A l g}$ & 1.1 & 2.5 & 4.3 & 6.7 & 10 & 15 & 23 & 40 & 90 \\
\hline $\log _{10}\left(m_{\mathrm{Gel}} / m_{\mathrm{Alg}}\right)$ & 0.041 & 0.398 & 0.633 & 0.826 & 1.00 & 1.18 & 1.37 & 1.60 & 1.95 \\
\hline$n_{\mathrm{Gel}} / n_{\mathrm{Alg}}$ & 8 & 18 & 30 & 47 & 70 & 105 & 162 & 281 & 632 \\
\hline$M_{\text {GelAlg. }} \cdot 10^{-6}, D$ & 0.75 & 1.25 & 1.89 & 2.75 & 3.93 & 5.71 & 8.57 & 14.6 & 32.5 \\
\hline$p H$ & \multicolumn{9}{|c|}{ Colloids; sols, if $\zeta$-potential values are showed; $\Delta \zeta= \pm 3.0 \mathrm{mV}$} \\
\hline 4.5 & \multicolumn{6}{|c|}{ high-viscous solutions } & -40.7 & -32.0 & +14.5 \\
\hline 4.0 & -34.1 & -39.8 & -22.7 & \multicolumn{4}{|c|}{ large dense floccules } & +17.0 & gels \\
\hline 3.5 & -37.8 & -34.9 & -26.2 & -20.3 & +8.7 & +11.6 & +17.4 & +29.1 & gels \\
\hline
\end{tabular}

As follows from the $\zeta$-potential (Table 1), the GelAlg complexes have negative charges, when $m_{\mathrm{Gel}} / m_{\mathrm{Alg}}$ ratio is low, and they have positive charges, when this ratio is high. By lowering the $\mathrm{pH}$ value of biopolymer solutions, the recharging point of resulted particles is gradually shifting in the direction of increasing the alginate concentration. Intersection points of curves $\zeta=\mathrm{f}\left\{\log _{10}\left(m_{\mathrm{Gel}} / m_{\mathrm{Alg}}\right)\right\}$ with values of $\zeta=0.0$, correspond to the composition of completely neutral colloids (Figure 6). In these conditions, the formation of neutral complexes GelAlgo, able to precipitate quickly, 
takes place. The sign inversion occurs at the $\log _{10}\left(m_{\mathrm{Gel}} / m_{\mathrm{Alg}}\right) \approx 1.7$ at the $\mathrm{pH}=4.5$ (Figure $\left.6, a\right)$, decreases to $\approx 1.1$ at the $\mathrm{pH}=4.0$ (Figure $6, b$ ) and is reduced to $\approx 0.9$ at the $\mathrm{pH}=3.5$ (Figure 6, $c$ ).

\section{Charge of large floccules, not measurable by electrophoresis}

It is problematically to study gelatine-alginate interaction at low $\mathrm{pH}$ value, because of precipitation of alginic acid (HAlg) and strong increasing positive charge of gelatine macromolecules. Moreover, the $\mathrm{pH}$ values lower than 3.0 are impossible to obtain by means of citric acid, and a small amount of $\mathrm{HCl}$ is necessary. In this condition, electrophoresis will be aggravated by electrolyze of $\mathrm{HCl}$ solution, resulting $\mathrm{H}_{2(\mathrm{Gas})}$ at cathode and $\mathrm{Cl}_{2(\mathrm{Gas})}$ at anode. This fact makes impossible to determine correct the zeta potential of colloid particles. The large floccules are formed quickly and are separated spontaneously from the supernatant solutions in many cases. Even in instances when floccules do not precipitate immediately and have been subjected to electrophoresis, they did not form a clearly defined borders so as it was shown in Figure 1. We cannot quantify the charge values in these conditions, but we can estimate the changes of charge sign due to the characteristic behaviour of colloidal suspensions [7]. Thus, after the stirring of obtained colloids about $20 \ldots 30$ minutes in each system was established an equilibrium. The system stability remains unchanged for the next several days (Figure 7).

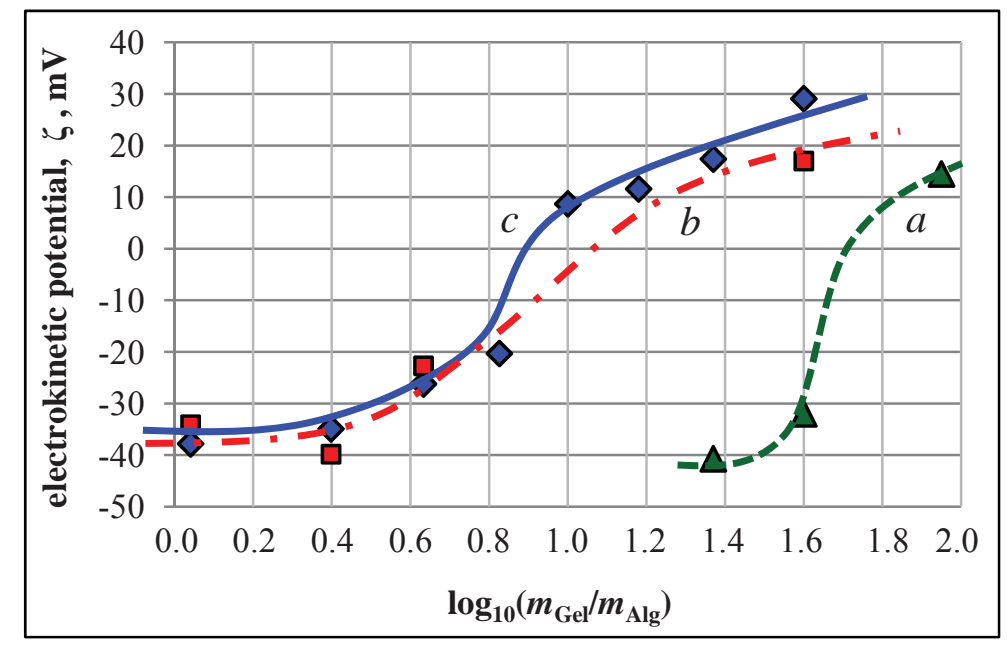

Figure 6. Dependence of electrokinetic potential $\zeta$ of the polyelectrolytes concentration ratio: a) at $\mathrm{pH}=4.5 ; \mathrm{b})$ at $\mathrm{pH}=4.0 ; \mathrm{c}$ ) at $\mathrm{pH}=3.5$.
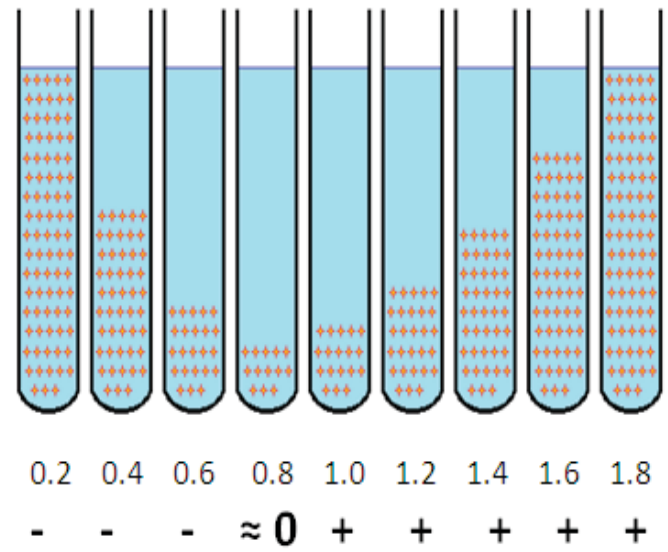

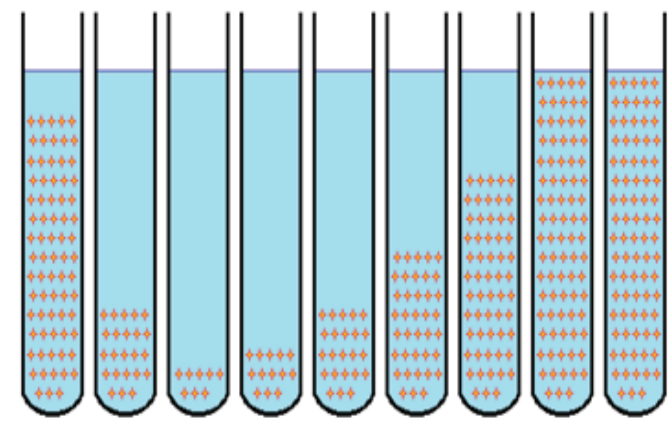

$\begin{array}{lllllllll}0.2 & 0.4 & 0.6 & 0.8 & 1.0 & 1.2 & 1.4 & 1.6 & 1.8\end{array}$

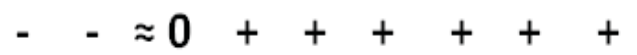

Figure 7. The sedimentation equilibrium aspect; $\log _{10}\left(m_{\mathrm{Gel}} / m_{\mathrm{Alg}}\right)$; sign of the floccules charge. Left for $\mathrm{pH}=\mathbf{3 . 0}$; right for $\mathrm{pH}=\mathbf{2 . 5}$.

The maximum density of floccules formed at $\mathrm{pH}=3.0$ corresponds to the range of concentration $m_{\mathrm{Gel}} / m_{\mathrm{Alg}}$ ratio from 4.0 to 10.0. In a more acidic medium $(\mathrm{pH}=2.5)$ the range of rapid deposition of dense flocks shifted towards lower values of $m_{\mathrm{Gel}} / m_{\mathrm{Alg}}$ ratio (from 2.0 to 7.0 ). At the same time, in each case the contents of both external vials remained 
in suspension. It is obvious that the formation and rapid deposition of large floccules testifies zero charge of GelAlg colloids [7]. Conversely, decreasing the deposition rate in excess of one polyelectrolyte indicates the presence of large quantities of stabilizing charges. Naturally, this charge is negative in the left edge of series, because of excess of alginate anions, and is positive in the right edge of series. At low $\mathrm{pH}$ values (3.0 and 2.5), the changes of floccules charge correspond to the $\zeta$-potential changes for particles of sols with high dispersion grade, obtained at $\mathrm{pH}>3.0$. During the increasing of $m_{\mathrm{Gel}} / m_{\mathrm{Alg}}$ ratio, the charges of GelAlg colloids became positive, similarly with changes shown in Table 1. Neutralization points, obtained using Figures 4 and 5, allow to estimate $n_{\text {Gel }} / n_{\text {Alg }}$ ratio by Eq.(4) and $M_{\text {GelAlg }}{ }^{0}$ for neutral 3D-colloids by Eq.(5) (Table 2).

Table 2

Parameters of neutral 3D-colloids GelAlgo.

\begin{tabular}{cccccc}
\hline $\boldsymbol{p} \boldsymbol{H}$ & 4.5 & 4.0 & 3.5 & 3.0 & 2.5 \\
\hline $\boldsymbol{l o g}_{\mathbf{1 0}}\left(\boldsymbol{m}_{\mathrm{Gel}} / \boldsymbol{m}_{\mathrm{Alg}}\right)$ & 1.7 & 1.1 & 0.9 & 0.80 & 0.60 \\
$\boldsymbol{m}_{\mathrm{Gel}} / \boldsymbol{m}_{\mathrm{Alg}}$ & 50.1 & 12.6 & 7.9 & 6.3 & 4.0 \\
$\boldsymbol{n}_{\mathrm{Gel}} / \boldsymbol{n}_{\mathrm{Alg}}$ & 352 & 89 & 56 & 44 & 28 \\
$\boldsymbol{M}_{\mathrm{GelAlg}} \cdot 10^{-6}, D$ & 18.2 & 4.86 & 3.18 & 2.61 & 1.79 \\
\hline
\end{tabular}

Results of calculations showed in the Table 2 demonstrate clearly that inversion of charge (neutral) point shifts towards smaller values of the $m_{\mathrm{Gel}} / m_{\mathrm{Alg}}$ ratio when $\mathrm{pH}$ became lower. In other words, at $\mathrm{pH}$ values approaching the IEP, even a small amount of polyuronic salts causes the coagulation of the proteins.

\section{Phase diagram construction and its applications for microcapsules}

The data obtained and discussed above can be extrapolated in visual interpretation of function $\mathrm{pH}-$ composition, resulting phase state diagram of the gelatine-alginate system (Figure 8).

The phase state diagram of gelatine-alginate at various $\mathrm{pH}$ values shows the phase phenomena occurring in the $\mathrm{pH}$ range of 2.0...6.0 at concentrations from $0.0 \%$ to $3.0 \%$ of gelatine and from $0.0 \%$ to $0.3 \%$ of alginate. These values correspond to the concentrations, used in the producing of beverages by means of protein-carbohydrate interaction [5], and to the conditions of microencapsulation by the method of complex coacervation $[4,18]$. Recall that at $\mathrm{pH}$ values more than IEP, gelatine is in form of negative-zwitterion, and is becoming "pure" anion at $\mathrm{pH}>10$. At the same time, gelatine is in form of positive charged zwitterion at $\mathrm{pH}$ lower than IEP, and is converted in "pure" cation, Gel', at $\mathrm{pH}<2.0[3,10]$.

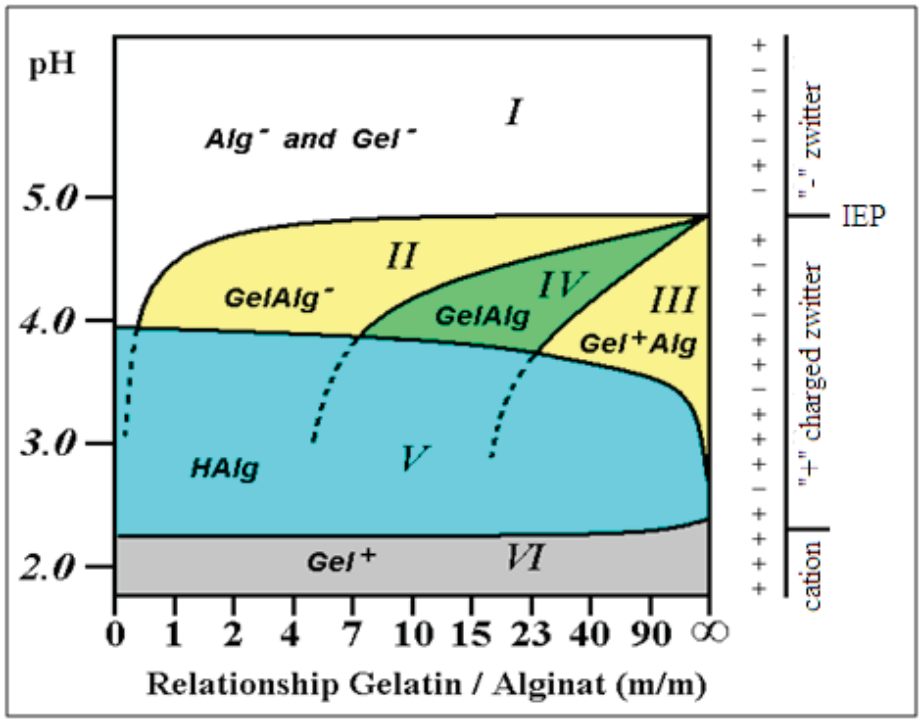

Figure 8. Phase diagram of the gelatine-alginate system.

The Region I corresponds to the mutual repulsion of negatively charged zwitterions $\mathrm{Gel}^{>+}$and "pure" anions of $\mathrm{Alg}$. Although the main part of the Region I is located at $\mathrm{pH}>5$ (above the isoelectric point of gelatine), it still comes down to a $\mathrm{pH}$ of $\approx 4.5$ in the left side, wherein the ratio $m_{\mathrm{Gel}} / m_{\mathrm{Alg}}$ is low. We explain this fact that at high concentrations 
of alginate occur full repayments of positive charges of gelatine by the excess of negative charges, as evidenced by the formation of transparent viscous solutions instead of sols and floccules in these conditions.

In the Region II, the formation of a high-negatively charged sols or suspensions formed from the large floccules takes place. In such colloidal systems the sedimentation does not occurs for several days. Deposition delay takes place because of powerful repulsion between surfaces of floccules. It is known that alginic or hyaluronic acid occurs in the anionic form at $\mathrm{pH}$ greater than $3.5[9,23]$. Due to this fact, there is a sufficient number of negative charges of alginate in this Region, which can neutralize many molecules of gelatine-zwitterion. Excess of negative charges, derived from alginate, is decisive for electrokinetic potential of the colloidal system in this Region, and total charge of resulting colloids $\left(\mathrm{GelAlg}^{-}\right)$are negative. Colloids, formed in this Region, can minimize the using of gelatine in production of wines for the consumers, which abstain from the proteins of animal origin [24]. Electrokinetic properties of so charged colloids can be similar to agent properties for the binding of metal ions [25], and in our opinion, can substitute these agents in the production of wines and beverages.

In the Region III, there is an interaction similar to that observed in the Region II: colloids are kinetic stable and do not precipitate quickly, i.e., large excess of the gelatine in the form of zwitterions causes the global charge of colloids to be positive. Therefore, we have named as $\mathrm{Gel}^{+} \mathrm{Alg}$ the floccules and sols obtained in these conditions.

The Region IV characterizes the formation of dense large flocks that undergo rapid sedimentation, which occurs in few minutes. The impossibility to determine their charge by electrophoresis proves that charges of sols and floccules, resulting in the Region IV, are close to zero. Numerically, the change of charge point is shifted to the higher gelatine concentrations, when $\mathrm{pH}$ increases (Table 1). Control experiments showed the greatest turbidity of pure gelatine solutions at $\mathrm{pH}$ 4.75-5.00. Therefore, the top point of this area corresponds to the isoelectric point of gelatine type "B" [12]. This fact explains nearly neutral charge of complexes GelAlg in the conditions, when $m_{\mathrm{Gel}} / m_{\mathrm{Alg}}$ ratio tends to infinite and $\mathrm{pH}$ is near IEP value. Moreover, the neutral flocks form the agglomerates, which quickly lose their ability to peptize. According to the basic knowledge of colloid chemistry, the conditions, corresponding to the Region IV, are of practically importance for the deposition of undesirable colloids in beverages by means of co-sedimentation mechanism [7]. Therefore, it can be argued that the formation of neutral 3D-structures GelAlg is not desirable in other cases, when the repulsion between the colloidal particles, but not their mutual coagulation, is necessary. Thus, it should be demonstrated that neutral charge of protein-polyuronate complexes GelAlg or GelHur is undesirable for stability of oil-containing microcapsules, possessing the shells, which are formed from these biopolymers.

In the Region $\mathbf{V}$ that is characterized by low $\mathrm{pH}$ values, the pattern is a somewhat peculiar. At $\mathrm{pH}<4$ gelatine solutions acquire high transparency, inherent for the true solutions of HMC and for non-colloids. This transparency appears because of straightening of the molecules due to the intra-molecular repulsion (excess of positive charges in the zwitterions $\mathrm{Gel}^{+{ }^{+}-}$) [6,7]. At the same time, the solutions of alginate in this $\mathrm{pH}$ range, behave quite differently. At $\mathrm{pH}$ values below 3.8, the alginate solutions lose their transparency and the alginate precipitates in form of alginic acid at $\mathrm{pH} \approx 3.5$, taking place a reaction: $\mathrm{Alg}^{-}+H^{+} \rightarrow H A l g$. Solutions of the hyaluronates behaved similarly: hyaluronic acid precipitates in the form of gelled sticks by lowering the $\mathrm{pH}$ below 3.5. Thus, in the Region $\mathbf{V}$ the formation of GelAlg or GelHur floccules is accompanied and is aggravated by the protonation of polyanions.

The above-described phase diagram "gelatine-alginate" explains well the behaviour and stability of lipidcontaining microcapsules with gelatine-polyuronate shells under various conditions. In the model solutions with $\mathrm{pH}$ corresponding to the Regions II...VI, microcapsules behave differently. In the conditions of Region II the microcapsules are high stable and do not stick (Figure 9, a). For neutral GelAlg ${ }^{0}$ or GelHur ${ }^{0}$ complexes, microcapsules suffer agglomeration (Figure 9, $b$ ) and total destruction (Figure 9, $c$ ).

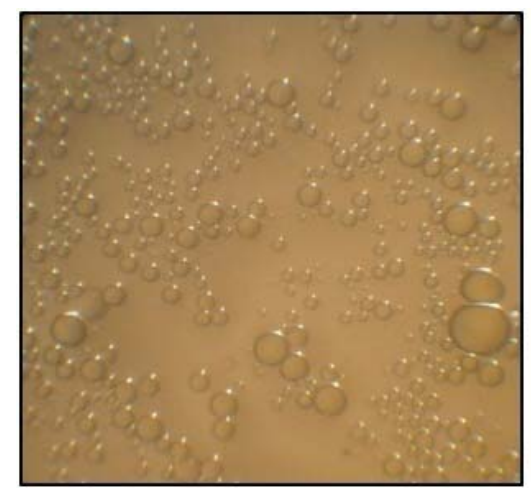

$a$

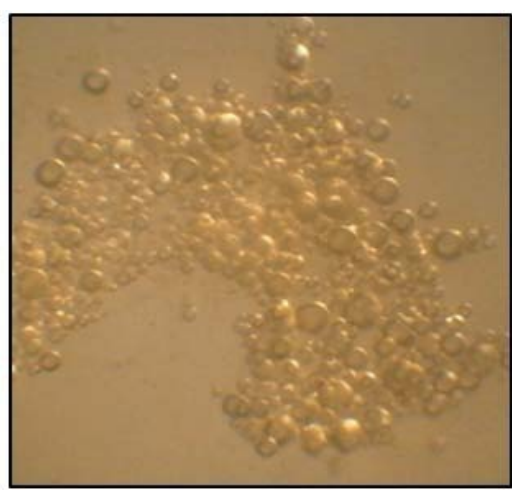

$b$

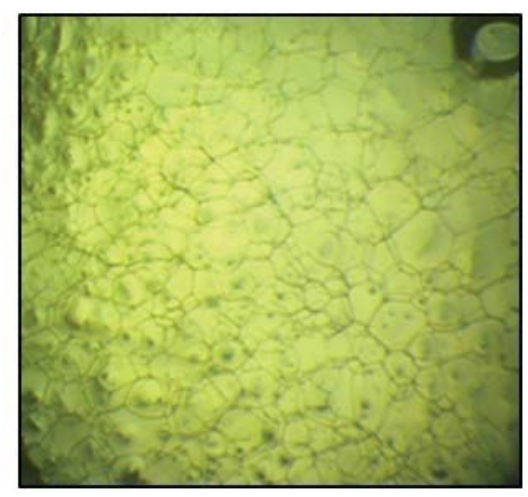

c

Figure 9. Microcapsules with gelatine-hyaluronic shells: a) stable, in the conditions of Region II; b) coagulated in the conditions of Regions V or VI; c) destroyed after 3 days in the conditions of Region VI. 
It can be noted that gelatine-alginate or gelatine-hyaluronate shells of microcapsules, being under the conditions of Region VI, undergo following reactions:

$$
\begin{aligned}
& \text { GelAlg }^{-}+H^{+} \rightarrow \text { GelAlg }^{0}(\text { or: GelHur } \\
&
\end{aligned}
$$

In our opinion, the complete destruction of high-molecular structures does not occur immediately. Only structural recombination of shells, described by Eq.(6) and Eq.(7), accompanied by a change of their charge and, obviously, with latent changes in the conformation of macromolecules, take place. Due to this fact, the destruction of microcapsules with removal of lipid phase completed after 3...4 days. Conglutination of microcapsules and recombination of their shells, occurring in Regions V and VI, are accompanied by the release of encapsulated content and with the formation of foamlike dodecahedron structures, consisting of protein-poliuronate colloids (Figure 9, c).

The regularities of formation of charged colloids, probably, also refer to protein-based colloids, which are formed with different polyuronic acids, pectins and other polysaccharides possessing acidic functional groups.

\section{Conclusions}

There were found the conditions for the formation of negatively charged, the positively charged and the neutral colloids. It was observed that the properties of protein/polyuronate system depend on their mass ratio logarithmically. The molecular weight of neutral charged colloids decreases with diminishing the $\mathrm{pH}$ value. A phase state diagram, which reflects the influence of $\mathrm{pH}$ and ratio of the components (gelatine and alginate) on electric charge and stability of the resulting polymer complexes, was developed. The Diagram is of great practical interest for predicting the properties of different food systems. In particular, the Diagram shows the most suitable conditions for obtaining of microcapsules with stable gelatine-alginate or gelatine-hyaluronate shells with a negative electric charge $\{$ Region $\mathrm{II}, \mathrm{pH}=3.0 \ldots 4.5$, $m_{\mathrm{Gel}} / m_{\mathrm{Alg}}$ ratio $2 \ldots 8$, GelAlg, $\left.M_{\mathrm{GelAlg}}=(1 \ldots 3) \cdot 10^{6} \mathrm{D}\right\}$. Due to their negative charge, the colloids, obtained in the Region II, can be used for sedimentation of different positively charged particles in different food systems, inclusive wines and beverages. The Region III, with strong positive charge of colloids $\left\{\mathrm{pH}=3.0 \ldots 4.0, m_{\mathrm{Gel}} / m_{\mathrm{Alg}}=40 \ldots 90, \mathrm{Gel}^{+} \mathrm{Alg}\right.$, $\left.M_{\text {GelAlg }}=(15 \ldots 35) \cdot 10^{6} D\right\}$, is suitable for microencapsulation, but is characterized by great consumption of gelatine for shell's constructing. Phase diagram shows that gelatine-alginate colloids with $\zeta \approx 0\{$ Region $\mathrm{IV}, \mathrm{pH}=3.0 \ldots 4.5$, $m_{\mathrm{Gel}} / m_{\mathrm{Alg}}$ ratio $\left.10 \ldots 30, \mathrm{GelAlg}^{0}, M_{\mathrm{GelAlg}}=(4 \ldots 10) \cdot 10^{6} \mathrm{D}\right\}$ are not suitable for microencapsulation. At the same time, the complexes with zero charge, obtained under the conditions of Region IV, are of greatest interest for quickly clarification of beverages.

\section{Acknowledgements}

This work is done within the framework of Research Project 15.817.02.30A ("Development of Methods and Techniques for Modernisation of Nuts (Juglans Regia L.) Processing Technology Using the Biologically Active Constituents in the Functional Foods), co-financed by the Academy of Sciences of Moldova and by the Technical University of Moldova.

\section{References}

1. Shinde, U.A.; Nagarsenker, M.S. Characterization of Gelatine-Sodium Alginate Complex Coacervation System. Indian Journal of Pharmaceutical Science, 2009, 71(3), pp. 313-317.

2. Belitz, H.-D.; Grosch, W.; Schieberle, P. Food Chemistry. Springer Verlag: Berlin, 2009, 1070 p.

3. Gómez-Guillén, M.C.; Giménez, B.; López-Caballero, M.E.; Montero, M.P. Functional and bioactive properties of collagen and gelatine from alternative sources. Food Hydrocolloids, 2011, 25, pp. 1813-1827.

4. Roy, A.; Bajpai, J.; Bajpai, A.K. Development of Calcium Alginate - Gelatine Based Microspheres for Controlled Release of Endosulfan as a Model Pesticide. Indian Journal of Chemical Technology, 2009, 16, pp. 388-395.

5. Aharnsilawat, T.; Pongsawatmanit, R.; McClements, D.J. Stabilization of Model Beverage Cloud Emulsions Using Protein-Polysaccharide Electrostatic Complexes Formed at the Oil-Water Interface. Journal of Agricultural Food Chemistry, 2006, 54(15), pp. 5540-5547.

6. Heinzmann G.; Tartsch, B. Alginates, chitosanes and xanthans. Characterization of food ingredients by GPC/SEC with triple detection. Agrofood Industry Hightech, 2009, 20(4), pp. 56-59.

7. Birdi, K.S. (editor). Handbook of Surface and Colloid Chemistry. Taylor \& Francis Group, 2016, 694 p.

8. Doublier, J.-L.; Garnier, C.; Renard, D.; Sanchez, C. Protein-polysaccharide interactions. Current Opinion in Colloid \& Interface Science, 2000, 5, pp. 202-214.

9. Lenormand, H.; Tranchepain, F.; Deschrevel, B.; Vincent, J.-C. The hyaluronan-protein complexes at low ionic 
strength: How the hyaluronidase activity is controlled by the bovine serum albumin. Matrix Biology, 2009, 28, pp. 365-372.

10. Steinbuchel, A.; Rhee, S.K. Polysaccharides and Polyamides in the Food Industry. Wiley-VCH: Weinheim. 2005, $783 \mathrm{p}$.

11. Hammad Umer; Hemlata Nigam; Asif M. Tamboli; M. Sundara Moorthi Nainar. Microencapsulation: Process, Techniques and Applications. International Journal of Researches in Pharmaceutical and Biomedical Sciences, 2011, 2 (2), pp. 474-481.

12. Pignatello, R. Biomaterials Applications for Nanomedicine. Intech: Rijeka, Croatia, 2011, 458 p.

13. Jusova, A.A.; Gusev, I.V.; Lipatova, I.M. Properties of hydro gels based on mixtures of sodium alginate with other polysaccharides of natural origin. Chemistry of vegetable raw materials, 2014, 4, pp. 59-66 (in Russian).

14. Jackson, C.R.; Raja, P.M.V.; Prakash, S. A Novel Electrophoretic Deposition Device: Effects of Alginate Viscosity Grade on Deposition Kinetics. Journal of Biotechnology \& Biomaterials, 2012, doi:10.4172/2155-952X.S6-002.

15. Khair, A.S.; Squires, T.M. The Influence of Hydrodynamic Slip on the Mobility of a Spherical Colloidal Particle. Physics of Fluids, 2009, 21(4), 042001. http://dx.doi.org/10.1063/1.3116664.

16. Dimova, O.; Baerle, A.; Tatarov, P.; Verejan, A. Process for producing alginates from brown algae. Republic of Moldova Patent, 2013, No. MD 669 (in Romanian).

17. Zadorojnâi; L. Process for obtaining the sodium hyaluronate, the hyaluronic acid and the protein-hyaluronic acid complex. Republic of Moldova Patent, 2006, No. MD 3099 (in Romanian).

18. Baerle, A.; Tatarov, P.; Dimova, O.; Cojohari, C. Process for microencapsulation of food and cosmetic oil compositions. Republic of Moldova Patent, 2012, No. MD 557 (in Romanian).

19. Masuelli, M.A. Mark-Houwink Parameters for Aqueous-Soluble Polymers and Biopolymers at Various Temperatures. Journal of Polymer and Biopolymer Physical Chemistry, 2014, 2(2), pp. 37-43.

20. Lima, A.M.F.; Soldi, V.; Borsali, R. Dynamic Light Scattering and Viscosimetry of Aqueous Solutions of Pectin, Sodium Alginate and their Mixtures. Effects of Added Salt, Concentration, Counterions, Temperature and Chelating Agent. Journal of Brazilian Chemical Society, 2009, 20(9), pp. 1705-1714.

21. Masuelli, M.A.; Illanes, C.O. Review of the characterization of sodium alginate by intrinsic viscosity measurements. Comparative analysis between conventional and single point methods. International Journal of Biomaterials Science and Engineering, 2014, 1(1), pp. 1-11.

22. Vold, I.M.N.; Kristiansen, K.A.; Christensen, B.E. The Mark-Houwink equation of alginates and chitosans. Biomacromolecules; Addition/Correction. 2007, 8(8), pp. 2627-2627.

23. Fukushima, M.; Tatsumi, K.; Wada, S. Evaluation of the Intrinsic Acid-Dissociation Constant of Alginic Acid by Considering the Electrostatic Effect. Analytical Sciences, 1999, 15, pp. 1153-1155.

24. Pyevich, C. Why is Wine so Fined? Vegetarian Journal, 1997, XVI(1), https://www.vrg.org/journal/vj97jan/971wine.htm.

25. Rinn, J.-C.; Robillard, B. Alginate or pectate gel deficient in gelling ions for use in binding metal ions. United States Patent, 1996, No. US 5567451. 